\title{
The Combination of Green Tea Extract and Eriodictyol Inhibited High-Fat/High-Sucrose Diet-Induced Cholesterol Upregulation Is Accompanied by Suppression of Cholesterol Synthesis Enzymes
}

\author{
Mai Yamashita, Motofumi Kumazoe, Yuki NaKamura, Yeong Seon Won, \\ Jaehoon BAE, Shuya YamashitA and Hirofumi TACHIBANA* \\ Division of Applied Biological Chemistry, Department of Bioscience and Biotechnology, \\ Faculty of Agriculture, Kyushu University, Fukuoka 812-8581, Japan
}

(Received February 18, 2016)

\begin{abstract}
Summary Western diets induce obesity associated with an increased risk of hypercholesterolaemia. Indeed, obesity-induced hypercholesterolaemia is correlated with increased coronary cardiovascular disease (CVD) risk. Male C57BL/6J mice were fed a normal diet, highfat and high-sucrose diet (HF/HS), HF/HS with green tea extract powder $\operatorname{diet}(\mathrm{HF} / \mathrm{HS}+\mathrm{GT})$, $\mathrm{HF} / \mathrm{HS}$ with eriodictyol diet (HF/HS+Eri), or HF/HS with green tea extract powder and eriodictyol diet (HF/HS+GT+Eri) for 8 wk. Body weight was lower in the HF/HS+GT+Eri group than in the HF/HS group $(-8.3 \%, p<0.01)$. The HF/HS diet elicited an upregulation of total cholesterol levels $(-63 \%, p<0.001)$, and low-density lipoprotein (LDL) levels $(-89 \%$, $p<0.001)$ were significantly suppressed by the GT +Eri diet. Conversely, no change $(p>0.05)$ was observed in the HF/HS+GT and $\mathrm{HF} / \mathrm{HS}+$ Eri groups. The HF/HS diet-induced hepatic mRNA increase in 3-hydroxy-3-methylglutaryl-coenzyme A reductase (HMGCR) was ameliorated $(-73 \%)$ by the oral administration of green tea extract and eriodictyol. Moreover, the GT+Eri diet suppressed HF/HS diet-induced upregulation of 3-hydroxy-3-methylglutaryl-coenzyme A synthase (HMGCS) $(-75 \%, p<0.05)$. Furthermore, the LDL receptor (LDLR) levels were higher in the HF/HS $+\mathrm{GT}+$ Eri group $(+50 \%, p<0.05)$ than in the $\mathrm{HF} /$ HS group. These results suggest that a combination of green tea and eriodictyol decreases cholesterol levels, particularly LDL levels, accompanied by the suppression of HMGCR and HMGCS levels and upregulation of LDLR levels in the liver.
\end{abstract}

Key Words green tea, eriodictyol, hypercholesterolemia, LDL, HMGCR

The Western diet includes high-fat and high-sugar foods, such as junk food, and is typical in many developed countries, particularly the United States. Thus, obesity is recognized as the most widespread nutritional disease in developed countries (1). The Western diet has been linked to metabolic syndrome accompanied by hypertension, obesity, and hypercholesterolemia $(2,3)$.

Cardiovascular disease (CVD) is the main cause of death in patients with metabolic syndrome. Hypercholesterolemia is related to an increase in CVD risk associated with metabolic syndrome. In particular, a high level of low-density lipoprotein (LDL) in the blood has a positive correlation with risk leading to CVD (4-6). Collectively, the downregulation of LDL levels is an attractive strategy for preventing CVD.

3-Hydroxy-3-methylglutaryl-coenzyme A reductase (HMGCR) is a rate-controlling enzyme in the cholesterol synthesis pathway (7) and is a well-known target for

\footnotetext{
*To whom correspondence should be addressed.

E-mail: tatibana@agr.kyushu-u.ac.jp

Abbreviations: ALT, alanine transaminase; AST, aspartate aminotransferase; EGCG, epigallocatechin-3-O-gallate; HDLC, high-density lipoprotein cholesterol; HMGCR, 3-hydroxy3-methylglutaryl-coenzyme A reductase; HMGCS, 3-hydroxy3-methylglutaryl-coenzyme A synthase; LDL-C, low-density lipoprotein cholesterol; LDLR, LDL receptor, TC, total cholesterol; VLDL-C, very low-density lipoprotein cholesterol.
}

hypercholesterolemia. Statins, including lovastatin, fluvastatin, and simvastatin, that target HMGCR are widely used to relieve patients with hypercholesterolemia. Although statins remain the major hypolipidemic drugs at present, the number of patients treated with statins has increased along with the number of those suffering from side effects or not responding well to therapy (7).

Low density lipoprotein receptor (LDLR) is a mosaic protein involved in clearance of cholesterol and cholesteryl ester-containing LDL particles from blood (8). The important function of the LDLR in the regulation of cholesterol is suggested by the 900 types of mutations found in patients with familial hypercholesterolemia. Considering LDLR mutation causes familial hypercholesterolemia with elevated blood levels of cholesterol and the early onset of atherosclerosis (9), LDLR is a crucial negative regulator in blood levels of cholesterol.

Tea (Camellia sinensis), one of the world's most popular beverages, is consumed worldwide, and its healthpromoting effects have been extensively investigated (10). In particular, green tea has various physiological benefits, such as an anti-obesity effect (11-14). An epidemiological analysis based on 82,365 Japanese individuals demonstrated that green tea consumption inversely correlated with CVD risk (15). Moreover, animal intervention studies have demonstrated that green tea and its extract exhibit hypocholesterolemic and hypo-LDL 


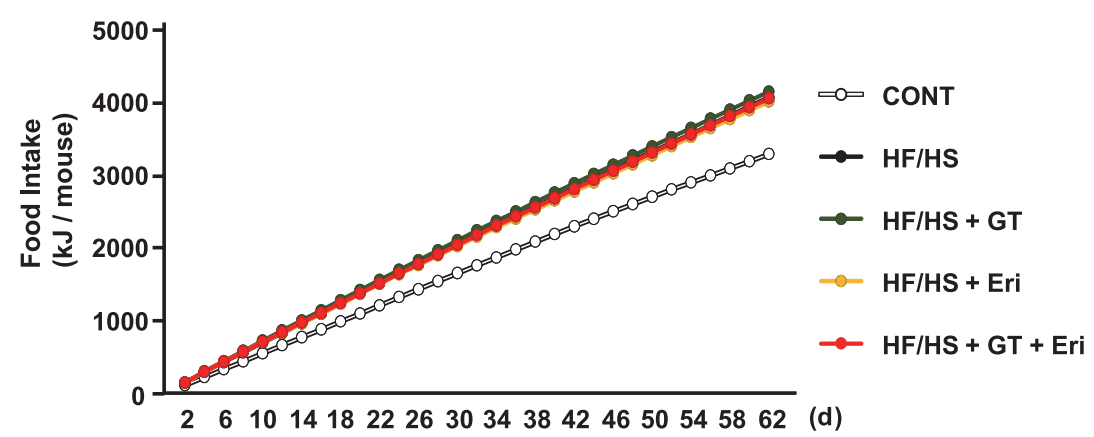

Fig. 1. Food Intake. Food intake was measured on a per cage basis throughout the study and represents cumulative energy intake.

effects $(16,17)$. Epigallocatechin-3-O-gallate (EGCG) is a major bioactive constituent in green tea and has hypocholesterolemic activity $(18,19)$. Catechins account for $>30 \%$ of the solids in a normal infusion of green tea, and EGCG is the most abundant, accounting for $58 \%$ of total catechins $(20,21)$.

The 67-kDa laminin receptor $(67 \mathrm{LR})$ is a non-integrin receptor for laminin with high affinity. Recent studies show 67LR mediates several effects of EGCG including anti-cancer (22), anti-inflammatory (23), neuroprotective (24) and immune reinforcement effects (25).

Eriodictyol is one of the major polyphenols in Citrus limon (lemons) and is an intermediate metabolite of the catechin synthesis pathway in $C$. sinensis. We previously performed the metabolic profiling of 43 green tea cultivar panels by liquid chromatography-mass spectrometry and chemometrics to evaluate molecules that contributed to the anti-cancer effects of green tea extract. We discovered that eriodictyol potentiated the effect of EGCG by amplifying EGCG-induced 67LR dependent signaling (26). Considering the high-affinity binding of EGCG to 67LR (Kd of $40 \mathrm{nM}$ ) (27) and the plasma concentration of EGCG $(<1 \mu \mathrm{M})$ in daily green tea intake, $67 \mathrm{LR}$ may play a central role in the various effect of EGCG. In this context, eriodictyol may potentiate other beneficial effects of EGCG including hypocholesterolemic activity.

Here we demonstrate the effect of a combination of green tea extract and eriodictyol on the hypocholesterolemic activity in mice fed a high-fat and high-sucrose (HF/HS) diet.

\section{MATERIALS AND METHODS}

Animals and diets. C57/BL6 mice (12 wk; male) were obtained from Kyudo (Saga, Japan) and were maintained with a 12-h-light-dark cycle (light from 8 am to $8 \mathrm{pm}$ ) in a humidity-controlled room. All animals engaged in this study were acclimated for $7 \mathrm{~d}$ and fed an AIN-93G diet. All diets were purchased from KBT (Tokyo, Japan) (Supplemental Table 1).

Animals were separated into indicated groups $(n=6$ per group): (1) a negative control group fed the AIN93G diet (CONT); (2) a control group fed a HF/HS diet (HF/HS); (3) a group fed a HF/HS diet containing $0.2 \%$ green tea extract powder $(\mathrm{HF} / \mathrm{HS}+\mathrm{GT})$; (4) a group fed a HF/HS diet containing $0.045 \%$ eriodictyol (HF/ HS + Eri); and (5) a group fed a HF/HS diet containing
$0.2 \%$ green tea extract powder and $0.045 \%$ eriodictyol (HF/HS + GT +Eri). The doses of both eriodictyol (28) and green tea extract (29) in our study were determined as reference.

Extracts of green tea were obtained from green tea leaves using seething water (over $70^{\circ} \mathrm{C}$, approximately $30 \mathrm{~min}$ ) and freeze-drying techniques as previously described (29). Analysis of the green tea extract was measured using HPLC (Supplemental Table 2) as previously described (29). Mouse body weight was assessed once a week. At the end of $8 \mathrm{wk}$ of feeding, all mice were sacrificed after $16 \mathrm{~h}$ of fasting. Plasma samples were obtained after centrifugation $(20 \mathrm{~min}, 2,000 \times \mathrm{g})$. Tissues (perirenal and epididymal depots) and organs were weighed. All animal studies were performed in accordance with the Japanese domestic law (protocol no. 105) and notification (protocol no. 6) of the Japanese government for the welfare of experimental animals and according to the guidelines for animal experiments at the Faculty of Agriculture, Kyushu University.

Biochemical analyses of plasma and liver samples. Plasma levels of aspartate aminotransferase (AST), alanine aminotransferase (ALT), total cholesterol (TC), high-density lipoprotein-cholesterol (HDL-C), LDL-cholesterol (LDL-C), and very LDL-C (VLDL-C) were measured using a Cholesterol E-Test Kit, the transaminase CII-Test Kits both from Wako Pure Chemical Industries, Ltd., Kyoto, Japan), and HDL-C and LDL-C/VLDL-C Quantification Kit (BioVision, Milpitas, CA), respectively. All kits were used according to the manufacturers' instructions.

Quantitative real-time polymerase chain reaction (PCR). cDNA was synthesized from total RNA ( $1 \mu \mathrm{g})$ using the PrimeScript RT Reagent Kit (Takara Bio, Tokyo, Japan). Gene expression was analyzed by quantitative real-time PCR using the SYBR green procedure and a Thermal Cycler DiceH Real-Time System (Bio-Rad, Hercules, CA). Primer sequences are provided in Supplemental Table 3. Beta-actin was chosen as an internal control to normalize the quantitative real-time PCR results.

Statistical analyses. All statistical analyses were performed using one-way ANOVA with a Tukey's test using GraphPad Prism software. Data are presented as means \pm SE. $p<0.05$ was considered significant.

\section{RESULTS}

The cumulative energy intake did not significantly 
Table 1. Effects of green tea extract and eriodictyol on accumulation of body fat and organs in HF/HS-fed C57BL/6J mice.

\begin{tabular}{lccccc}
\hline & CONT & HF/HS & HF/HS + GT & HF/HS + Eri & HF/HS + GT + Eri \\
\hline Final body weight, g & $28.28 \pm 0.24^{\mathrm{a}}$ & $39.90 \pm 0.43^{\mathrm{b}}$ & $37.83 \pm 0.76^{\mathrm{bc}}$ & $38.82 \pm 0.81^{\mathrm{bc}}$ & $36.63 \pm 0.58^{\mathrm{c}}$ \\
Perirenal fat, g & $0.15 \pm 0.01^{\mathrm{a}}$ & $0.74 \pm 0.03^{\mathrm{b}}$ & $0.66 \pm 0.02^{\mathrm{b}}$ & $0.70 \pm 0.05^{\mathrm{b}}$ & $0.64 \pm 0.02^{\mathrm{b}}$ \\
Epididymal fat, g & $0.53 \pm 0.04^{\mathrm{a}}$ & $2.00 \pm 0.09^{\mathrm{b}}$ & $1.88 \pm 0.06^{\mathrm{b}}$ & $1.85 \pm 0.12^{\mathrm{b}}$ & $1.77 \pm 0.04^{\mathrm{b}}$ \\
Liver, g & $0.99 \pm 0.02^{\mathrm{ab}}$ & $1.03 \pm 0.01^{\mathrm{a}}$ & $0.95 \pm 0.01^{\mathrm{b}}$ & $0.98 \pm 0.02^{\mathrm{ab}}$ & $0.98 \pm 0.02^{\mathrm{ab}}$ \\
Quadriceps muscle, g & $0.45 \pm 0.01^{\mathrm{a}}$ & $0.45 \pm 0.01^{\mathrm{a}}$ & $0.44 \pm 0.01^{\mathrm{a}}$ & $0.46 \pm 0.01^{\mathrm{a}}$ & $0.45 \pm 0.01^{\mathrm{a}}$ \\
\hline
\end{tabular}

Results are the means \pm SE $(n=6)$. Different letters (a,b, and c) indicate significant difference $(p<0.05)$ by Tukey's test.

A

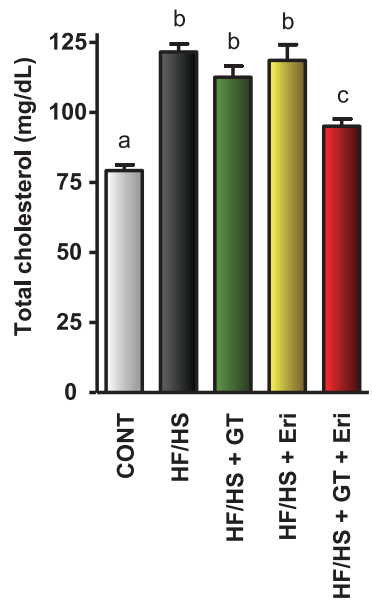

D

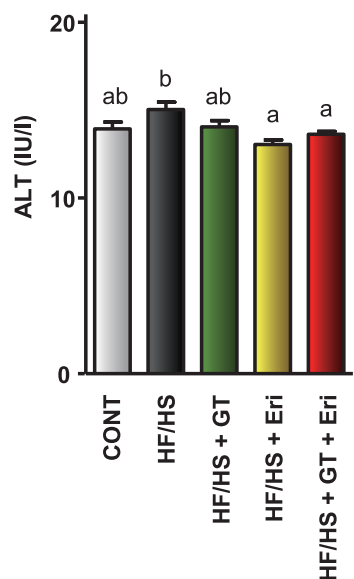

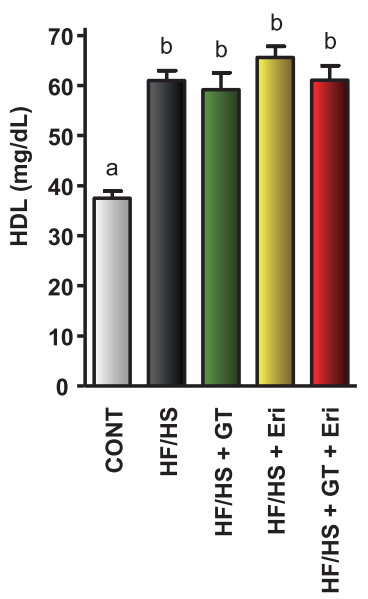

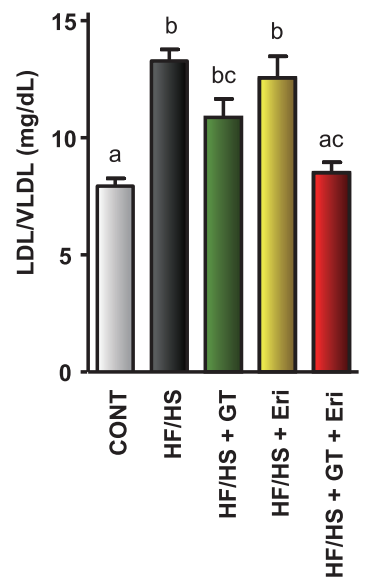

E

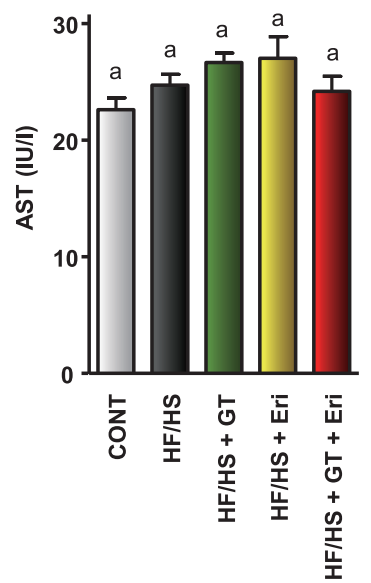

Fig. 2. Effects of green tea extract and eriodictyol on serum levels of total cholesterol, HDL, LDL/VLDL, ALT, AST in HF/ HS-diet-fed mice. Plasma total cholesterol (A), HDL (B), LDL/VLDL (C), ALT (D), and AST (E) levels at the end of treatment period. Results are presented as means \pm SE $(n=6)$. Values bearing different lowercase letters $(\mathrm{a}, \mathrm{b}$, and $\mathrm{c})$ are significantly different $(p<0.05)$ by Tukey's test.

differ among the HF/HS groups (HF/HS, HF/HS+GT, $\mathrm{HF} / \mathrm{HS}+\mathrm{Eri}$, and HF/HS+GT+Eri) (Fig. 1). The HF/HS groups had more body weight gain $(p<0.001)$ than the CONT group (Table 1). The HF/HS + GT+Eri diet significantly attenuated the HF/HS diet-induced body weight increase $(p<0.01)$. In contrast, consistent with a previous report (29), the HF/HS + GT (0.2\%) group showed no significant difference in body weight compared with the HF/HS group ( $p>0.05)$. The same result was obtained in the HF/HS+Eri group. Compared with the CONT group, all HF/HS diets substantially increased perirenal and epididymal fat weights. The $\mathrm{HF} / \mathrm{HS}+\mathrm{GT}+$ Eri group had a moderately decreased weight of perirenal and epididymal fat compared with the HF/HS group. The HF/ HS group had slightly higher liver weight gain than the CONT group, and HF/HS+GT diet attenuated the HF/ HS diet-induced liver weight increase (Table 1). There was no significant difference for any group in the weight of the quadriceps muscle.

HF/HS diet intake significantly enhanced the plasma 
A

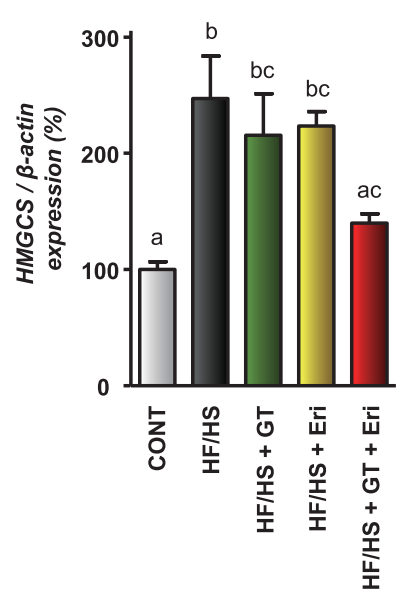

B

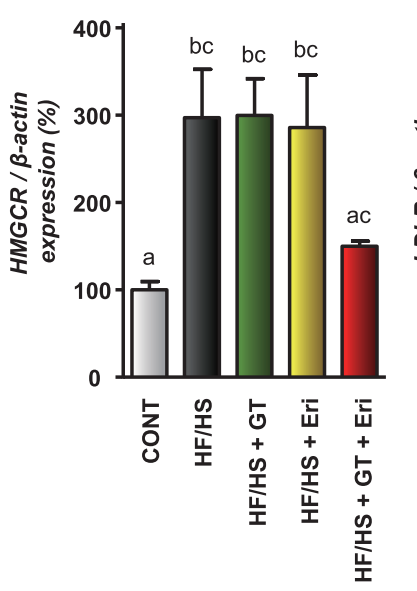

Fig. 3. The mRNA expression levels of cholesterol-related genes including HMGCS (A), HMGCR (B), and LDLR (C) in the liver of mice fed HF/HS diets containing green tea extract and eriodictyol for $8 \mathrm{wk}$. All gene expressions were determined by quantitative real-time PCR. Results represent means \pm SE $(n=6)$. Values bearing different lowercase letters $(\mathrm{a}, \mathrm{b}$, and $\mathrm{c})$ are significantly different $(p<0.05)$ by a Tukey's test.

levels of TC, HDL-C, LDL-C, and VLDL-C (Fig. 2A-C). We previously reported that $0.2 \%$ GT derived from standard cultivar intake did not affect the cholesterolemic markers, including TC, LDL-C, and VLDL-C levels, in the same HF/HS diet-induced hypercholesterolaemia model (29). Similarly, GT intake alone could not attenuate HF/ HS diet-induced upregulation of cholesterolemic markers, including TC, LDL-C, and VLDL-C levels $(p>0.05)$. The same result was obtained for eriodictyol treatment alone. In contrast, the HF/HS+GT+Eri diet significantly decreased the levels of TC $(p<0.001)$, LDL-C, and VLDL-C $(p<0.001)$ compared with those for the HF/ HS diet. In particular, considering LDL-C levels, which are crucially correlated with CVD risk, GT+Eri in combination decreased $89 \%$ of HF/HS diet-induced LDL-C level upregulation. In contrast, there was no significant difference in plasma HDL-C levels for an HF/HS group (HF/HS, HF/HS+GT, HF/HS+Eri, and HF/HS+GT+Eri) $(p>0.05)$. In contrast, eriodictyol could not potentiate the suppressive effect of green tea extract on the HF/HS diet-induced triglyceride increase (Supplemental Fig. 1). Although the HF/HS group exhibited slightly increased plasma ALT levels compared with CONT group, the groups fed HF/HS+Eri $(p<0.01)$ and $\mathrm{HF} / \mathrm{HS}+\mathrm{GT}+$ Eri $(p<0.05)$ diets had markedly decreased ALT levels compared with the HF/HS group (Fig. 2D). There was no significant difference in plasma AST levels for any group $(p>0.05)$ (Fig. 2E).

The mRNA expression of hepatic genes that regulate cholesterol biosynthesis and cholesterol acquisition are shown in Fig. 3. HMGCR and HMGCS, the key enzymes in the mevalonate pathway, play a crucial role in cholesterol biosynthesis. The mRNA levels of these key cholesterol biosynthesis enzymes were highly upregulated in HF/HS-diet-fed mice compared with those in the normal diet-fed mice $(p<0.05)$. As previously reported (29), $0.2 \%$ green tea extract alone could not suppress $\mathrm{HF} /$ HS diet-induced upregulation of HMGCR and HGMCS
mRNA levels. The same results were obtained in the HF/ HS+Eri group. Strikingly, in the presence of eriodictyol, $0.2 \%$ green tea extract intake drastically decreased the mRNA expression of HMGCS $(p<0.05)$ compared with the HF/HS group (Fig. 3A). Moreover, there was no significant difference in HMGCR mRNA levels between the CONT group and HF/HS + GT+Eri group ( $p>0.05)$ (Fig. 3B). We also confirmed there is correlation between HMGCR and serum LDL/VLDL levels (Supplemental Figure 2). The HF/HS+GT+Eri diet significantly increased the expression of the LDL receptor (LDLR), which plays a crucial role in the uptake of cholesterol and known LDL-negative regulators compared with the HF/HS diet $(p<0.05)$ (Fig. 3C). Taken together, GT/Eriodictyol in combination has a potent effect on HF/HS-diet induced cholesterol increase.

\section{DISCUSSION}

The main cause of the death in patients with metabolic syndrome is CVD. In this respect, hypercholesterolemia-induced arteriosclerosis plays a crucial role in the progression of CVD. Previous studies have reported oxidative damage as an important etiologic factor in atherosclerosis. In particular, according to the oxidative stress theory, oxidative modification of LDL is believed to play a key role in the development of atherosclerosis $(30,31)$. Oxidized LDLs are taken up by arterial wall cells, particularly macrophages, in a non-regulated manner through the scavenger receptor pathway. This process leads to the formation of foam cells, the hallmark of arteriosclerosis lesions (32). Therefore, decreasing plasma LDL levels is considered to be an important therapeutic approach.

There have been many epidemiologic studies on the effect of green tea on CVD $(33,34)$. Onakpoyaa et al. reported that dietary supplementation with green tea generates modest decreases in LDL-C levels (35). In this study, we demonstrated that the combination of 
green tea and eriodictyol significantly decreased plasma cholesterol levels, particularly LDL-C levels in HF/ HS-diet-fed mice. However, green tea intake alone did not decrease plasma cholesterol or LDL-C levels. These results suggest that eriodictyol potentiates the hypocholesterolemic effect of green tea.

To assess the hypocholesterolemic effect of this combination, we evaluated the impact of GT+Eri on the expression of cholesterol-related enzymes. Our results show that eriodictyol strongly potentiates the suppressive effect of green tea on HMGCR and HMGCS. Importantly, HMGCR is a restrictive enzyme in the cholesterol synthesis pathway and a well-known drug target for hypercholesterolemia treatment. A previous report showed that a high-dose administration of $1 \%$ green tea extract powder significantly suppressed hepatic HMGCR and HMGCS expressions in mice fed a HF/HS diet, whereas $0.2 \%$ green tea extract powder intake alone could not induce the hypocholesterolemic effect (29). Our data also shows that a $0.2 \%$ GT diet alone did not have any suppressive effect on the expression of either HGMCR or HMGCS. In contrast, under the supplementation with eriodictyol, the low dose of GT intake dramatically decreased HF/HS diet-induced HMGCR and HMGCS upregulation by $75 \%$ and $70 \%$, respectively.

Kuhn et al. (36) and Bursill and Roach (37) demonstrated that a high concentration of EGCG (approximately 50-200 $\mu \mathrm{M}$ ), the main catechin of green tea, increased the expression of LDLR in HepG2 cells. Considering the plasma concentration of EGCG, the effect of EGCG alone is thought to be limited. In this study, compared with the HF/HS group, GT intake alone did not show an increase in LDLR levels, but GT+Eri intake significantly potentiated the levels. Thus, many studies have reported the hypocholesterolemic effect of EGCG and green tea extract at high concentrations, and it is difficult to take such a high quantity of green tea in the average daily diet.

Considering the limited hypocholesterolemic effect of green tea extract alone, it is important to screen the compounds that potentiate its effect. However, there are few available candidates that amplify the hypocholesterolemic effect of green tea extract, partly because there is no established screening approach.

We previously demonstrated metabolic profiling-based chemical-mining using 43 green tea extract panels and identified eriodictyol, a polyphenol compound that potentiated the apoptosis-inducing potency of EGCG against multiple myeloma cells (26). Importantly, based on this screening approach, we succeed in uncovering the compounds that drastically enhanced the hypocholesterolemic effect of green tea extract in vivo. These findings suggest that the metabolic profiling-based apoptosis-inducing potency of EGCG against multiple myeloma cells may be a useful approach to screen the compounds that potentiate the beneficial effect of EGCG.

Our recent study showed that eriodictyol potentiated the protein kinase B (Akt)-activation effect of EGCG for anticancer activity (26). These findings suggest that Akt activation is involved in the combined effect of green tea and eriodictyol in hypocholesterolemia. The combination of green tea and eriodictyol did not show a significant difference in the weight of perirenal or epididymal fat. Green tea has various anti-obesity effects, such as neutral fat accumulation-inhibiting action and energy consumption-promoting action (11). Our results suggest that the combined effect of green tea and eriodictyol on hypocholesterolemia is involved in this antiobesity function.

Statins, inhibitors of HMGCR, are used in patients with hypercholesterolemia worldwide. However, statin treatment sometimes induces serious adverse effects, such as hepatic dysfunction and rhabdomyolysis $(38,39)$. Green tea is one of the most popular beverages worldwide, and many studies have reported the safety of green tea (4042). A previous study reported that an extraordinarily high green tea concentration is demanded to induce hepatotoxicity in rats and mice (43). In addition, stability in the blood and safety of eriodictyol, which is abundantly present in citrus fruit peels, has also been shown (44). In this study, there were no significant differences for any group in plasma AST levels, an index of hepatotoxicity. Moreover, the HF/HS+Eri and HF/HS+GT+Eri diets significantly decreased the plasma ALT levels compared with the HF/HS diet. These results support that the combination of green tea and eriodictyol did not result in hepatotoxicity.

Currently, pharmacological inhibition of HMGCR (statins) is among the current regimens for hypercholesterolemia (45). Furthermore, ezetimibe is a comparatively new hypocholesterolemic agent that suppresses the absorption of biliary and dietary cholesterol in the intestine (46). In contrast, we revealed that the combination of green tea extract powder and eriodictyol improved hypercholesterolemia, decreased the expression levels of hepatic cholesterol synthase enzymes, including HMGCR and HMGCS, and increased the expression level of LDLR in mice fed with a HF/HS diet. Although further research is required to establish how eriodictyol potentiates the hypocholesterolemic effect of green tea, our results suggest that an unexpected combination of green tea and eriodictyol, which are very abundant food factors, drastically decreased LDL-C expression by unique mechanisms through suppression of HF/HS-diet induced HMGCR/HGMCS expression and induction of LDLR upregulation. Taken together, green tea supplemented with eriodictyol could be used to decrease serum LDL levels.

\section{Funding}

This research was supposed by grants from the Project of the NARO Bio-oriented Technology Research Advancement Institution (Integrated research for agriculture and interdisciplinary fields). This work was supported in part by JSPS Kakenhi (grant nos.: 22228002 and $15 \mathrm{H} 02448$ to H. Tachibana), a Grant-in-Aid for JSPS Fellows to M. K. (PD), and JSPS Kakenhi (grant no.: 15K18821). The funders had no role in the study design, data collection or analysis, decision to publish, or preparation of the manuscript. 


\section{Author contributions}

M. Y. and M. K. designed the study and drafted the manuscript. M.Y., M. K., Y. N., W. Y. S., J. B., and S. Y. performed the experiments and $\mathrm{H}$. T. conducted whole research. All authors reviewed the manuscript and helped in the study design.

\section{REFERENCES}

1) Shen W, Chuang CC, Martinez K, Reid T, Brown JM, Xi L, Hixson L, Hopkins R, Starnes J, McIntosh M. 2013. Conjugated linoleic acid reduces adiposity and increases markers of browning and inflammation in white adipose tissue of mice. J Lipid Res 54: 909-922.

2) Chen YK, Cheung C, Reuhl KR, Liu AB, Lee MJ, Lu YP, Yang CS. 2011. Effects of green tea polyphenol (-)-epigallocatechin-3-gallate on newly developed high-fat/ western-style diet-induced obesity and metabolic syndrome in mice. J Agric Food Chem 59: 11862-11871.

3) Despres JP, Lemieux I. 2006. Abdominal obesity and metabolic syndrome. Nature 444: 881-887.

4) Cromwell WC, Otvos JD, Keyes MJ, Pencina MJ, Sullivan L, Vasan RS, Wilson PW, D’Agostino RB. 2007. LDL particle number and risk of future cardiovascular disease in the Framingham Offspring Study - Implications for LDL management. J Clin Lipidol 1: 583-592.

5) Pyorala K, Pedersen TR, Kjekshus J, Faergeman O, Olsson AG, Thorgeirsson G. 1997. Cholesterol lowering with simvastatin improves prognosis of diabetic patients with coronary heart disease. Diabetes Care 20: 614-620.

6) Howard BV, Robbins DC, Sievers ML, Lee ET, Rhoades D, Devereux RB, Cowan LD, Gray RS, Welty TK, Go OT, Howard WJ. 2000. LDL cholesterol as a strong predictor of coronary heart disease in diabetic individuals with insulin resistance and low LDL. Arterioscler Thromb Vaso Biol 20: 830-835.

7) He WS, Jia CS, Yang YB, Ma Y, Zhang XM, Feng B, Jin J. 2011. Cholesterol-lowering effects of plant steryl and stanyl laurate by oral administration in mice. J Agric Food Chem 59: 5093-5099.

8) Gent J, Braakman I. 2004. Low-density lipoprotein receptor structure and folding. Cell Mol Life Sci 61: 2461-2470.

9) Hobbs HH, Russell DW, Brown MS, Goldstein JL. 1990. The LDL receptor locus in familial hypercholesterolemia: mutational analysis of a membrane protein. Аnпu Rev Genet 24: 133-170.

10) Lin CL, Huang HC, Lin JK. 2007. Theaflavins attenuate hepatic lipid accumulation through activating AMPK in human HepG2 cells. J Lipid Res 48: 2334-2343.

11) Sae-Tan S, Grove KA, Lambert JD. 2010. Laboratory studies on weight control and prevention of metabolic syndrome by green tea. Pharmacol Res 64: 146-154.

12) Lu Y, Hajifathalian K, Ezzati M, Woodward M, Rimm EB, Danaei G. 2014. Metabolic mediators of the effects of body-mass index, overweight, and obesity on coronary heart disease and stroke: a pooled analysis of 97 prospective cohorts with 1.8 million participants. Lancet 383: 970-983.

13) Rains TM, Agarwal S, Maki KC. 2011. Antiobesity effects of green tea catechins: a mechanistic review. J Nutr Biochem 22: 1-7.

14) Lu C, Zhu W, Shen CL, Gao W. 2012. Green tea polyphenols reduce body weight in rats by modulating obesityrelated genes. PLoS One 7: e38332.

15) Kokubo Y, Iso H, Saito I, Yamagishi K, Yatsuya H,
Ishihara J, Inoue M, Tsugane S. 2013. The impact of green tea and coffee consumption on the reduced risk of stroke incidence in Japanese population: the Japan public health center-based study cohort. Stroke $\mathbf{4 4}$ : 1369-1374.

16) Kim YJ, Houng SJ, Kim JH, Kim YR, Ji HG, Lee SJ. 2012. Nanoemulsified green tea extract shows improved hypocholesterolemic effects in C57BL/ 6 mice. J Nutr Biochem 23: 186-191.

17) Bursill CA, Abbey M, Roach PD. 2007. A green tea extract lowers plasma cholesterol by inhibiting cholesterol synthesis and upregulating the LDL receptor in the cholesterol-fed rabbit. Atherosclerosis 193: 86-93.

18) Mielgo-Ayuso J, Barrenechea L, Alcorta P, Larrarte E, Margareto J, Labayen I. 2014. Effects of dietary supplementation with epigallocatechin-3-gallate on weight loss, energy homeostasis, cardiometabolic risk factors and liver function in obese women: randomised, doubleblind, placebo-controlled clinical trial. Br J Nutr 111: 1263-1271.

19) Goto T, Saito Y, Morikawa K, Kanamaru Y, Nagaoka S. 2012. Epigallocatechin gallate changes mRNA expression level of genes involved in cholesterol metabolism in hepatocytes. Br J Nutr 107: 769-773.

20) Muramatsu K, Fukuyo M, Hara Y. 1986. Effect of green tea catechins on plasma cholesterol level in cholesterolfed rats. J Nutr Sci Vitaminol 32: 613-622.

21) Harbowy EM, Balentine AD. 1997. Tea chemistry. Crit Rev Plant Sci 16: 415-480.

22) Tsukamoto S, Huang Y, Umeda D, Yamada S, Yamashita S, Kumazoe M, Kim Y, Murata M, Yamada K, Tachibana H. 2014. 67-kDa Laminin receptor-dependent protein phosphatase 2A (PP2A) activation elicits melanomaspecific antitumor activity overcoming drug resistance. J Biol Chem 289: 32671-32681.

23) Byun EH, Omura T, Yamada K, Tachibana H. 2011. Green tea polyphenol epigallocatechin-3-gallate inhibits TLR2 signaling induced by peptidoglycan through the polyphenol sensing molecule 67-kDa laminin receptor. FEBS Lett 585: 814-820.

24) Gundimeda U, McNeill TH, Fan TK, Deng R, Rayudu D, Chen Z, Cadenas E, Gopalakrishna R. 2014. Green tea catechins potentiate the neuritogenic action of brainderived neurotrophic factor: role of 67-kDa laminin receptor and hydrogen peroxide. Biochem Biophys Res Commun 445: 218-224.

25) Santilli G, Piotrowska I, Cantilena S, Chayka O, D'Alicarnasso M, Morgenstern DA, Himoudi N, Pearson K, Anderson J, Thrasher AJ, Sala A. 2013. Polyphenon E enhances the antitumor immune response in neuroblastoma by inactivating myeloid suppressor cells. Clin Cancer Res 19: 1116-1125.

26) Kumazoe M, Fujimura Y, Hidaka S, Kim Y, Murayama K, Takai M, Huang Y, Yamashita S, Murata M, Miura D, Wariishi H, Maeda-Yamamoto M, Tachibana, H. 2015. Metabolic profiling-based data-mining for an effective chemical combination to induce apoptosis of cancer cells. Sci Rep 10: srep09474.

27) Tachibana H, Koga K, Fujimura Y, Yamada K. 2004. A receptor for green tea polyphenol EGCG. Nat Struct Mol Biol 11: 380-381.

28) Yoo JM, Kim JH, Park SJ, Kang YJ, Kim TJ. 2012. Inhibitory effect of eriodictyol on IgE/Ag-induced type I hypersensitivity. Biosci Biotechnol Biochem 76: 1285-1290.

29) Suzuki T, Kumazoe M, Kim Y, Yamashita S, Nakahara 
K, Tsukamoto S, Sasaki M, Hagihara T, Tsurudome Y, Huang Y, Maeda-Yamamoto M, Shinoda Y, Yamaguchi W, Yamada K, Tachibana H. 2013. Green tea extract containing a highly absorbent catechin prevents dietinduced lipid metabolism disorder. Sci Rep 3: 1-7.

30) Wiztum JL, Steinbeg D. 1991. Role of oxidized lowdensity lipoprotein in atherogenesis. J Clin Invest 88: 1785-1792.

31) Yla-Herttuala S, Palinski W, Rosenfeld ME, Parthasarathy S, Carew TE, Butler S, Wiztum JL, Steinbeg D. 1989. Evidence for the presence of oxidatively modified low density lipoprotein in atherosclerotic lesions of rabbit and man. J Clin Invest 84: 1086-1095.

32) Berrougui H, Cloutier M, Isabelle M, Khalil A. 2006. Phenolic-extract from argan oil (Argania spinosa L.) inhibits human low-density lipoprotein (LDL) oxidation and enhances cholesterol efflux from human THP-1 macrophages. Atherosclerosis 184: 389-396.

33) Moore RJ, Jackson KG, Minihane AM. 2009. Green tea (Camellia sinensis) catechins and vascular function. $\mathrm{Br}$ J Nutr 102: 1790-1802.

34) Hartley L, Flowers N, Holmes J, Clarke A, Stranges S, Hooper L, Rees K. 2013. Green and black tea for the primary prevention of cardiovascular disease. Cochrane Database Syst Rev 6: CD009934.

35) Onakpoyaa E, Spencera C, Heneghan C, Thompson M. 2014. The effect of green tea on blood pressure and lipid profile: A systematic review and meta-analysis of randomized clinical trials. Nutr Metab Cardiovasc Dis 24: 823-836.

36) Kuhn DJ, Burns AC, Kazi A, Dou QP. 2004. Direct inhibition of the ubiquitin-proteasome pathway by ester bond-containing green tea polyphenols is associated with increased expression of sterol regulatory elementbinding protein 2 and LDL receptor. Biochim Biophys Acta 1682: 1-10.

37) Bursill CA, Roach PD. 2006. Modulation of cholesterol metabolism by the green tea polyphenol (-)-epigallocatechin gallate in cultured human liver (HepG2) cells. J Agric Food Chem 54: 1621-1626.

38) Hansen KE, Hildebrand JP, Ferguson EE, Stein JH. 2005.

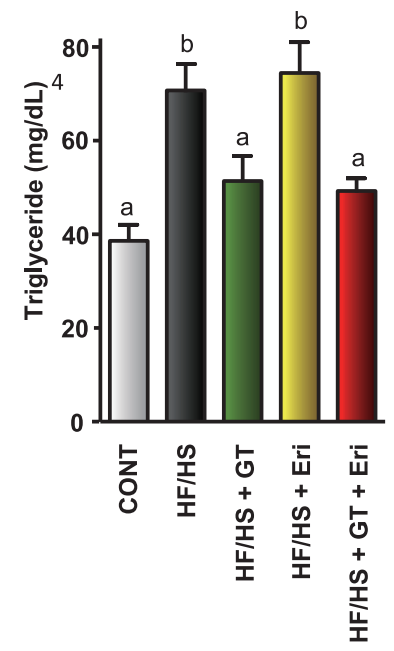

Supplemental Fig. 1. Effects of green tea extract and eriodictyol on serum levels of Triglyceride. Plasma Triglyceride at the end of treatment period. Results are the means \pm SE $(n=6)$. Values bearing different lowercase letters $(\mathrm{a}, \mathrm{b})$ are significantly different $(p<0.05)$ by Tukey's test. 5.
Outcomes in 45 patients with statin-associated myopathy. Arch Intern Med 165: 2671-2676.

39) Ballantyne CM, Corsini A, Davidson MH, Holdaas H, Jacobson TA, Leitersdorf E, Marz W, Reckless JP, Stein EA. 2003. Risk for myopathy with statin therapy in high-risk patients. Arch Intern Med 163: 553-564.

40) Sarma DN, Barrett ML, Chavez ML, Gardiner P, Ko R, Mahady GB, Marles RJ, Pellicore LS, Giancaspro GI, Low DT. 2008. Safety of green tea extracts: a systematic review by the US Pharmacopeia. Drug Saf 31: 469-484.

41) Chow HH, Cai Y, Hakim IA, Crowell JA, Shahi F, Brooks CA, Dorr RT, Hara Y, Alberts DS. 2003. Pharmacokinetics and safety of green tea polyphenols after multipledose administration of epigallocatechin gallate and polyphenon E in healty individuals. Clin Cancer Res 9: 3312-3319.

42) Hara Y. 1997. Influence of tea catechins on the digestive tract. J Cell Biochem Suppl 27: 52-58.

43) Chan PC, Ramot Y, Malarkey DE, Blackshear P, Kissling GE, Travlos G, Nyska A. 2010. Fourteen-week toxicity study of green tea extract in rats and mice. Toxicol Pathol 38: 1070-1084.

44) Miyake Y, Sakurai C, Usuda M, Fukumoto S, Hiramitsu M, Sakaida K, Osawa T, Kondo K. 2006. Difference in plasma metabolite concentration after ingestion of lemon flavonoids and their aglycones in humans. J Nutr Sci Vitaminol 52: 54-60.

45) Grundy SM. 1988. HMG-CoA reductase inhibitors for treatment of hypercholesterolemia. N Engl J Med 319: 24-33.

46) Ara R, Tumur I, Pandor A, Duenas A, Williams R, Wilkinson A, Paisley S, Chilcott J. 2008. Ezetimibe for the treatment of hypercholesterolaemia: a systematic review and economic evaluation. Health Technol Assess 12: $1-212$.

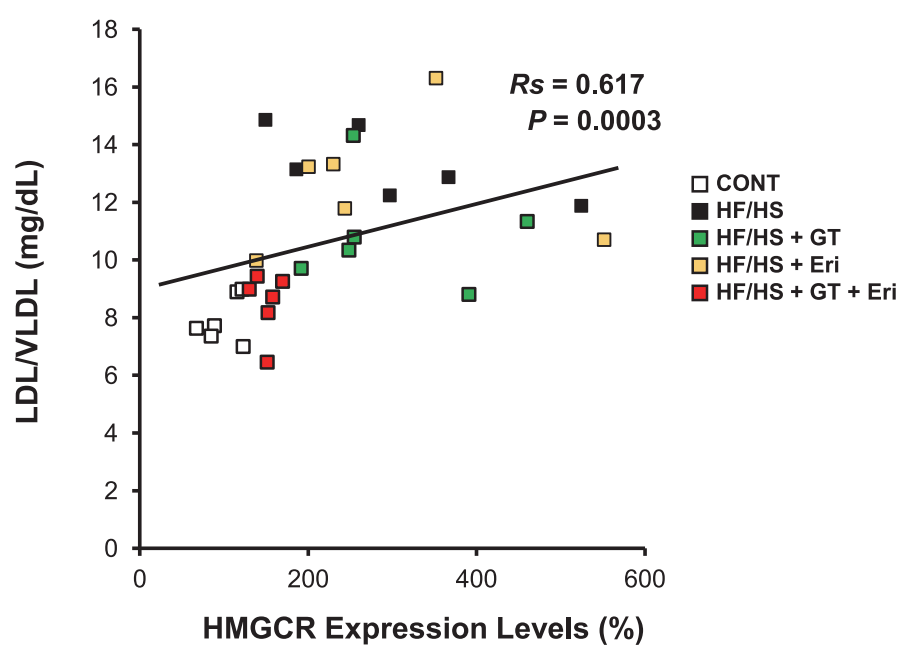

Supplemental Fig. 2. Correlation between HMGCR expression. All data were analyzed using Spearman's rank-correlation coefficient test. 
Supplemental Table 1. Consumption of experimental diets.

\begin{tabular}{|c|c|c|c|c|c|}
\hline & Control & $\mathrm{HF} / \mathrm{HS}$ & $\begin{array}{c}\mathrm{HF} / \mathrm{HS} \\
+\mathrm{GT}\end{array}$ & $\begin{array}{c}\mathrm{HF} / \mathrm{HS} \\
+\mathrm{Eri}\end{array}$ & $\begin{array}{c}\mathrm{HF} / \mathrm{HS} \\
+\mathrm{GT}+\mathrm{Eri}\end{array}$ \\
\hline \multicolumn{6}{|l|}{ Macronutrient composition } \\
\hline Protein, $\%$ of energy & 17.9 & 17.3 & 17.3 & 17.3 & 17.3 \\
\hline Fat, \% of energy & 7 & 54.8 & 54.8 & 54.8 & 54.8 \\
\hline Energy, MJ/kg & 15.8 & 21.1 & 21.1 & 21.1 & 21.1 \\
\hline \multicolumn{6}{|l|}{ Ingredient $(\mathrm{g} / \mathrm{kg})$} \\
\hline Vitamin $\operatorname{mix}^{1}$ & 10 & 10 & 10 & 10 & 10 \\
\hline Mineral mix ${ }^{2}$ & 35 & 35 & 35 & 35 & 35 \\
\hline Choline bitartrate & 2.5 & 2.5 & 2.5 & 2.5 & 2.5 \\
\hline L-Cystin & 3 & 3.8 & 3.8 & 3.8 & 3.8 \\
\hline Soybean oil & 70 & 20 & 20 & 20 & 20 \\
\hline Tertiary butylhydroquinone & 0.01 & 0.06 & 0.06 & 0.06 & 0.06 \\
\hline Sucrose & 100 & 200 & 200 & 200 & 200 \\
\hline Casein & 200 & 250 & 250 & 250 & 250 \\
\hline Cornstarch & 397.5 & 148.7 & 148.7 & 148.7 & 148.7 \\
\hline Pregelatinized cornstarch & 132 & 0 & 0 & 0 & 0 \\
\hline Cellulose & 50 & 50 & 48 & 50 & 48 \\
\hline Tallow & 0 & 140 & 140 & 140 & 140 \\
\hline Lard & 0 & 140 & 140 & 140 & 140 \\
\hline Green tea extract powder & 0 & 0 & 2 & 0 & 2 \\
\hline Eriodictyol & 0 & 0 & 0 & 0.45 & 0.45 \\
\hline
\end{tabular}

${ }^{1}$ Containing the following ( $\mathrm{g} / \mathrm{kg}$ vitamin mix): all-trans-retinol acetate, 0.80 ; cholecalciferol, 0.25 ; all-rac- $\alpha$-tocopherol acetate, 15; D-biotin, 0.20; cyanocobalamin (0.1\%), 2.5; folic acid, 0.20; Ca-panthothenate, 1.6; niacin, 3.0; pyridoxineHCI, 0.70; thiamin-HCl, 0.60; riboflavin, 0.60; phylloquinone, 7.5; and sucrose, 974.66.

${ }^{2}$ Containing the following ( $\mathrm{g} / \mathrm{kg}$ mineral mix): magnesium oxide, 24 ; calcium carbonate, 357; potassium phosphate monobasic, 250; tripotassium citrate monohydrate, 28; sodium chloride, 74; potassium sulfate, 46.6; iron citrate, 6.06; zinc carbonate, 1.65; manganese carbonate, 0.63; copper carbonate basic, 0.324; potassium Iodate, 0.01; sodium selenate, 0.01; ammonium molybdate $4 \mathrm{H}_{2} \mathrm{O}, 0.008$; sodium silicate $\cdot 9 \mathrm{H}_{2} \mathrm{O}, 0.0145$; chromium potassium sulfate $\cdot 12 \mathrm{H}_{2} \mathrm{O}, 0.275$; lithium chloride, 0.0174; boric acid, 0.0815; sodium fluoride, 0.0635; nickel carbonate basic $4 \mathrm{H}_{2} \mathrm{O}, 0.0306$; ammonium metavanadate, 0.0066; and sucrose, 209.78.

Supplemental Table 2. Composition of tea extract powders (mg/g).

\begin{tabular}{lc}
\hline & Green tea extract powder $(\mathrm{mg} / \mathrm{g})$ \\
\hline Galocatechin (GC) & 27.3 \\
(-)-Epigallocatechin (EGC) & 68.6 \\
Catechin (C) & 13.7 \\
(-)-Epicatechin (EC) & 20.9 \\
(-)-Epigallocatechin-3-gallate (EGCG) & 119.0 \\
Gallocatechin gallate (GCG) & 27.3 \\
(-)-Epicatechin-3-gallate (ECG) & 21.6 \\
Catechin gallate (CG) & 3.4 \\
Total catechins & 301.9 \\
\hline
\end{tabular}

Supplemental Table 3. Realtime PCR primers.

\begin{tabular}{lll}
\hline \multicolumn{1}{c}{ Gene } & \multicolumn{1}{c}{ Forward primer } & \multicolumn{1}{c}{ Reverse primer } \\
\hline HMGCS & 5'-GCCGTGAACTGGGTCGAA-3' & 5'-GCATATATAGCAATGTCTCCTGCAA-3' $^{\prime}$ \\
HMGCR & 5'-ATTCTGGCAGTCAGTGGGAACT-3' & 5'-CCTCGTCCTTCGATCCAATTTA-3' $^{\prime}$ \\
LDLR & 5'-CAGTCCCAGGCAGCGTTAT-3' & 5'-TTGATCTTGGCGGGTGTT-3' $^{\prime}$ \\
ACTB & 5'-CATCCGTAAAGACCTCTATGCCAA-3' & 5'-ATGGAGCCACCGATCCACA-3' \\
\hline
\end{tabular}

HMGCS, 3-hydroxy-3-methyl-glutaryl coenzyme A synthase; HMGCR, 3-hydroxy-3-methyl-glutaryl coenzyme A reductase; LDLR, low density lipoprotein receptor. 